\title{
Tactile feedback in a tele-operation pick-and-place task improves perceived workload
}

\author{
Thomas Baker ${ }^{1}$ and Matthias Rolf ${ }^{1}$ \\ Oxford Brookes University, UK \\ $\{18098352$, mrolf $\} @$ brookes.ac.uk
}

\begin{abstract}
Robotic tele-operation systems have vast potential in areas ranging from surgical robotics and underwater exploration to disposing of toxic, explosive and nuclear materials. While visual camera feeds for the human operator are typically available and well studied, tactile sensory information is often vital for successful and efficient manipulation. Previous studies have largely focused on execution time alone as measure of success of feedback methods on individual tasks. The present study complements this by a comparative analysis of vibration and visual feedback of tactile information across a range of manipulation tasks. Results show a significant reduction in perceived workload with the implementation of vibration feedback and an improvement of error rates for visual feedback. Contrary to expectation, we did not find a reduction in task completion time. The negative finding on completion time challenges the belief that the mere existence of task-relevant feedback aids efficient task completion. The reduced workload, however, clearly points out potential for enhancing performance on more difficult and prolonged tasks with highly skilled operators.
\end{abstract}

Keywords: Tele-operation · Tactile feedback · User study · Humanoid

\section{Introduction}

Tele-operation robotic systems are often defined as "operation of a system or machine at a distance" [1]. Tele-operation allows for direct access to inaccessible and hazardous environments [2] such as handling and disposing of toxic [3], explosive [4] and nuclear [5] material, and during space [6] and underwater exploration [7]. A tele-operation system that provides the operator with sensory feedback is referred to as a 'human-in-loop' system, where sensory feedback collected in the remote environment is provided and acted upon remotely. This is often referred to under the umbrella term of 'haptic' feedback although can be categorised into two main groups; cutaneous (force) and kinesthetic (pressure or tactile) feedback. For a system to provide haptic feedback it must provide substantial information from both cutaneous and kinesthetic systems.

How sensory data is provided to the operator falls under three main subcategories; force, vibration and cue systems. Force feedback being the restriction of movement usually through a device featuring mechanical input [8]. Vibration 
feedback (or vibrotactile) is the use of vibration to replicate touch, interactions with environments and contact information to an operator [9]. Vibration feedback has been extensively developed for virtual reality applications, in turn reducing the cost of small vibration actuators and becoming increasingly accessible and more appealing for such remote applications. Cues systems are made up of two main subcategories; Auditory (AF) [6] and Visual systems (VF) [10], where direct haptic feedback is substituted for graphical or audio cues to portray information about the remote environment [7].

There is extensive research that looks into sensory feedback methods within tele-operation applications, with studies ranging from the impact of visual cue systems within space exploration [6] to vibration feedback within underwater tele-robotics [7], in which time of completion and specific metrics relating to the particular scenario remain the focus of the analysis. An example of such assessment methods can be seen in [11] where average completion times is the main focus of analysis within a tele-operated maze task, the paper then adds the additional assessment metric of success rate within the scope of stacking rings on a peg. [12] looked at the tele-operation system usability by implementing a System Usability Scale (SUS), which asked users to rate 10 questions on a 7 point likert scale ranging from "strongly disagree" to "strongly agree". The questions covered different aspects of the tele-operation system, such as complexity, consistency and cumbersomeness.

Although the majority of the studies detailed are complex, such as RMIS (Remote-Assisted Minimally Invasive Surgery), the specificity of the respective tasks allow very little conclusion about general tasks involving, for example, pick and place. Furthermore, few research studies look to assess the impact of tactile sensory feedback across multiple manipulation tasks within a predefined study layout, assessing impact of the tactile feedback methods on multiple metrics. Whilst the time of completion is important, it should not be the sole parameter to measure the impact of a feedback method on a tele-operation system.

\subsection{Approach and Outline}

This paper tackles the empirical issues of tactile feedback in tele-operation by assessing tele-operation across a variety of increasingly sophisticated pick-andplace tasks on the Baxter humanoid robot with 24 previously untrained participants. The study goes beyond previous empirical investigations by comparing two different methods of tactile feedback not just based on time-to-completion, but holistically through addition of error rates and perceived workload (NASATLX), and across tasks. NASA-TLX [13] is a widely used assessment tool that measures perceived workload of a particular task, gained by measuring the global workload across six subscales: mental demand, physical demand, temporal demand, effort, frustration and performance.

The contribution of this paper are nuanced empirical findings that clearly challenge the dominating role of time measurement in current empirical methods: while finding no significant speed-up of task completion, both a reduced mental workload for vibro-tactile feedback and a reduced error-rate for visual 


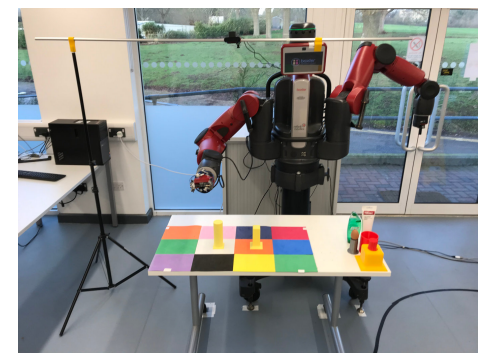

(a) Robot

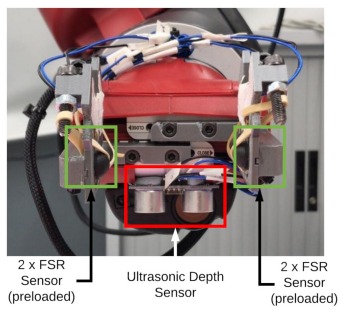

(c) Sensor placement on robot gripper

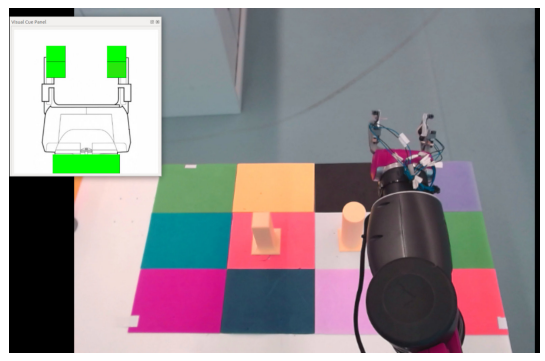

(b) Camera feed with visualized sensors.

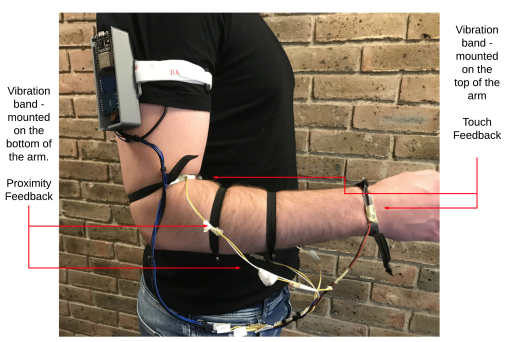

(d) Vibration band mounting

Fig. 1: Tele-operation system

feedback indicate possibly different usage scenarios and allow us to derive recommendations both for future practical applications as well as study designs. We describe our tele-operation system and feedback methods in Sec. 2, leading to the experimental design (Sec. 3) of three manipulation tasks. Results on time-to-completion, error rate, and workload are reported in Sec. 4. Possible limitations and implication or the results are discussed in Sec. 5 before giving recommendations and concluding remarks in Sec. 6 .

\section{Tele-operation system}

Rethink Robotics Baxter was used for the tele-operation task, a semi humanoid research robot that features a torso, 2 DOF head and two arms with 7 DOF joints, integrated camera, sonar sensors, torque sensors, collision avoidance and force sensing actuators (FSA) [14]. Due to the length of the arms, $104 \mathrm{~cm}$ from the shoulder joint to the end effector, Baxter is able to complete a range of tasks whilst maintaining accuracy and precision.

The key to an operator being able to see the environment they are teleoperating in is an efficient video feed (also known as a camera feed). A single camera was used and mounted above the remote enviroment. By only providing a single camera and in turn reducing the depth perception it was possible to ensure participants reliance on the feedback methods. 
HTC Vive was used as the tracking solution for the tele-operation system, a requirement for the tele-operation system is having accurate position and orientation tracking of the operator. This system was implemented using openVR, an API that allows for the development of virtual reality applications and serves as an interface between the VR hardware and software. By using unoffical python bindings it was possible to gain access to functions such as controller position and orientation, button presses and advanced options such as haptic pulses. A previously developed ROS implementation by [15] was advanced upon to allow a Baxter teleoperation system that used the in built inverse kinematics functionally to provide the operator with accurate tele-operation.

Piezoresistive force sensing resistors (FSR's) were used on the inside of the gripper's two fingers (Fig. 1.c) for detecting contact with objects in manipulation due to their sensitivity level, accessibility and simple implementation. Although in order to gain useful information with regards to object interactions in a remote environment the 'onset' (or first contact) to such objects is imperative. 'Onset' refers to the small interactions that occur before grasping the object itself. The standard FSR sensor did not allow for this discrete sensory information. A simple design that uses tensioned rubber bands set on a rubber pad was developed, this allowed for information relating to discrete object interactions to be gained. While the FSR sensors allowed for sensory information in regards to contact surfaces and object interactions, there was no information in relation to the proximity within the remote environment, more specifically depth perception. Ultrasonic sensors (HY-SRF05 [16]) were chosen as an appropriate choice of sensor to combat the issue, ultrasonic sensors being an instrument that measure distance using ultrasonic waves. The ultrasonic sensor was mounted on the underside of the gripper (Fig. 1.c) to provide the operator with useful information in relation to the distances in the remote environment.

Several alternatives are available to feed the sensor readings back to the user, the two detailed within this study are vibration feedback and visual cues. A novel system was developed within this study consisting of vibration bands located at multiple locations on the operators arm. These vibration bands use a disc motor mounted on a metal plate, secured using an elastic nylon material around the arm. The metal plate acting to amplify the vibration by changing the axis of rotation of the disk motor. This novel approach was developed as issues were encountered with both the internal and external mounting of vibration motors on the Vive controllers themselves, the two main issue being intermittent haptic pulses being present on the Vive controller along with a large amount of drift within the tracking solution itself because of noise on the accelerometer and infra red tracking, due to externally mounted vibration motors.

The final implemented system mounts two of the vibration bands on the top of the operators arm and two on the underside, these corresponding to the sensory information from the touch sensors (gripper) and the proximity sensor (ultrasonic). 

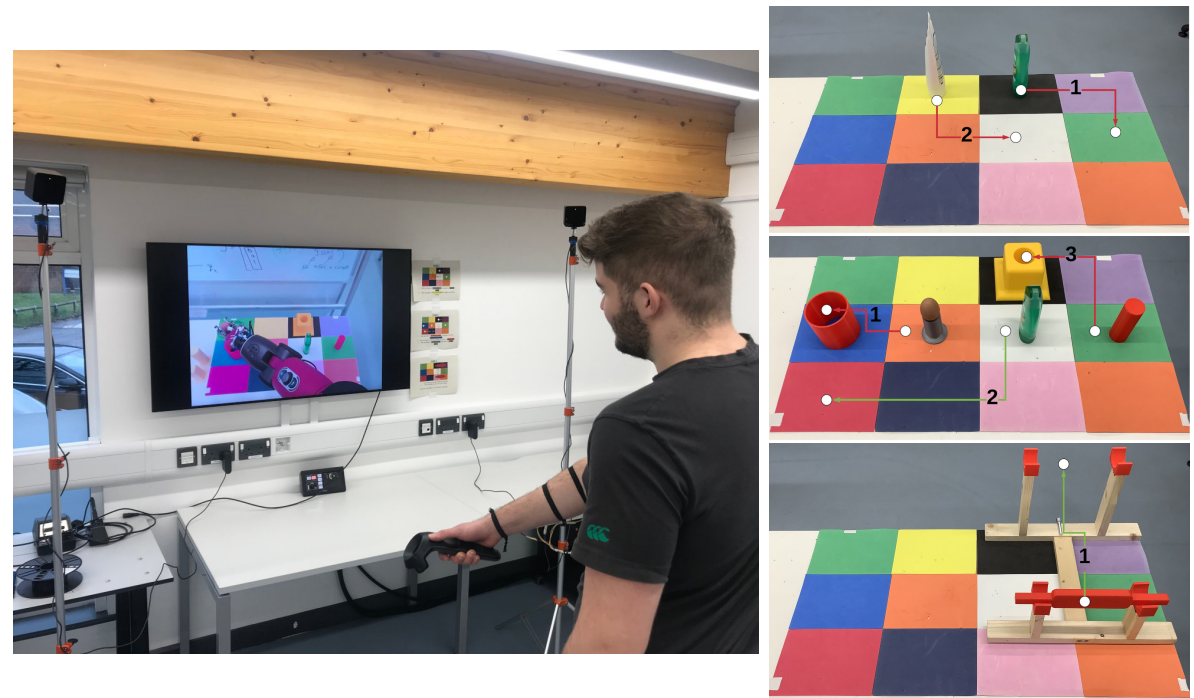

Fig. 2: (Left) User with controller in front of screen. (Right) Tasks 1-3 (from top): participants were asked to move the objects in a given order to a target tiles or place inside another structure (paths highlighted) by tele-operating Baxter.

\section{Experimental Methodology}

In order to get accurate and reliable results from the study it was imperative to have a clearly defined test scenario. A board with zoned areas was developed, allowing objects to be moved and manipulated within a measurable space allowing consistently same size areas. The tele-operation scenario was based on three individual tasks, that were designed to become increasingly challenging, starting with a very straight forward pick and place task, a slightly more advanced pick and place task on multiple levels, and ending on a complex parrellel bar task requiring a high level of accuracy. Both platforms of the final parrellel bar task holding the bar in a semicircle, allowing the bar to rotate with a small force applied, in turn increasing the level of complexity. The tasks were required to be general, real would manipulation tasks, interacting with day to day objects. This allowed for no formal training as participants would not be undertaking a specific task with unfamiliar objects or unknown operations.

The three tasks were undertaken in a fixed order with each task being completed three times with the same feedback method. Due to this fixed task ordering along with three feedback method conditions (no feedback, visual feedback and vibration feedback) there were 6 permutations required in relation to feedback ordering. Participants were randomly assigned into one of the six permutations, and assignments counter-balanced. For example, exactly four participants would do task 1 with visual feedback, task 2 with no feedback, and task three with vibration feedback; exactly four others are assigned to the other five permu- 
tations of feedback methods. Undertaking the three tasks with feedback analysis took between an hour and an hour and a quarter per participant. This meant that the feedback systems could be assessed in relation to the multiple measurable metrics - completion time, error rate and perceived workload. Providing a greater understanding and insight of the impact the feedback methods have would be gained.

Task objects were varied in relation to size, weight, shape, surface material and appearance. Specific objects were selected with large amounts of deformation to add complexity. Task 1 (Fig. 2, right top) was the initial task for each participant to undertake; simply picking up and moving object objects from two specific areas. Task 2 (Fig. 2, right middle) was slightly more advanced and required a higher level of accuracy and understanding of the remote environment. A rubber egg had to be dropped into a container, requiring a sense of depth, and a pole place in a stand. Task 3 (Fig. 2, right bottom) was the most complex and challenging manipulation task that the study required. Participants were required to move a parallel bar from one platform to another, requiring a sense of depth for the initial grasping and fine control of orientation.

Following a full set of three runs on each task, the NASA-TLX (Task Load Index) [13] was used to analyse the perceived workload of each task and the impact of associated feedback method. The NASA-TLX is a widely used assessment tool that measures the perceived workload of a particular task [13]. This is gained by measuring the global workload across six subscales: mental demand, physical demand, temporal demand,effort, frustration and performance.

The study was made up of 24 participants ( 17 male, 7 female) with ages ranging from 21 to $62(\mathrm{M}=27.70, \mathrm{SD}=10.72) .24$ participants being investigated as it allowed for each permutation of feedback ordering to be balanced. Participants level of self-assessed technological competence was measured (between 0 and 100) varying between 35 and $95(\mathrm{M}=65.83, \mathrm{SD}=17.17)$. Knowledge of robotics and tele-operation systems (also self assessed), measured between 0 and 100 and ranging from 5 and $90(\mathrm{M}=37.70, \mathrm{SD}=27.18)$. The study used participants with mainly dominant right hands, with a split of right $=18$, and left $=4$.

\section{Results}

Results on the overall time to completion for each run of the three tasks are shown in Fig. 3. Median times are consistently lower for the third attempt of each task. A statistical sign test against the Null-hypothesis of equal medians confirms the significance of this finding (Task $1 \mathrm{p}=0.064$, Task $2 \mathrm{p}=0.023$, Task $3 \mathrm{p}=0.015$ ). Contrary to the clearly pronounced learning curve, no significant difference are found between different feedback methods. A full breakdown for all tasks and feedback methods is shown in Fig. 4. No consistent pattern emerges in terms of advantage of any feedback method. Existing numeric differences were found insignificant by t-tests for all feedback comparisons on all tasks.

Error rates from object knock-overs and object-drops where 1.88 per run for no-feedback, 1.22 for visual feedback, and 1.53 for vibration feedback. A t-test 


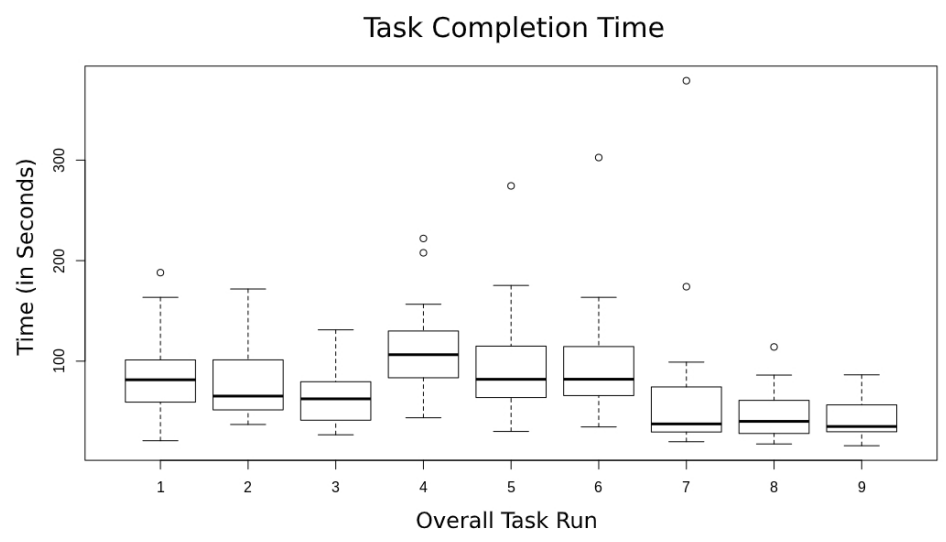

Fig. 3: Task completion times over tasks 1-3 with three runs each. A clear learning curve can be observed within each block of three, representing three consecutive runs on the same task.

showed a significant margin $(\mathrm{p}=0.003)$ between no-feedback and visual-feedback, but not vibration feedback $(\mathrm{p}=0.18)$. The $\mathrm{t}$-test, however, operates based on a Gaussian distribution assumption that was not confirmed valid on the data (Shapiro-Wilk test rejects Gaussian hypothesis with $\mathrm{p}<0.0001$ ). On the other hand, the parameter-free Kolmogrov-Smirnov test backs up the improvement from no-feedback to visual-feedback at least on trend-level $(\mathrm{p}=0.088)$.

Significant findings are shown in relation to the perceived workload of the tele-operation task, with vibration significantly reducing the perceived workload across all tasks, in comparison to both no feedback and visual cue conditions (see Fig. 5). With 22 out of 24 participants demonstrating a lower perceived workload with the addition of vibration feedback compared to no feedback and 18 out of 24 participants demonstrating a lower perceived workload with the addition of vibration feedback compared to visual cues, showing significant findings with binomial test $\mathrm{p}$ values of $3.588 \mathrm{e}-05$ for vibration feedback $<$ no feedback and 0.02266 for vibration feedback < visual cues. No significant results presented in relation to visual cues reducing the perceived workload with the tele-operation task. More in depth analysis was undertaken looking into frustration levels, extracted from the NASA-TLX data. This showed no significant reduction in frustration levels with the addition of both tactile sensory feedback methods.

\section{Discussion}

As is evident across all three tasks, neither the vibration feedback or visual cues have a positive impact on the overall completion time of tasks during teleoperation, this rejects the original hypothesis set out within the study. Although this finding is contrary to the hypothesis it is not an isolated case, studies such as 


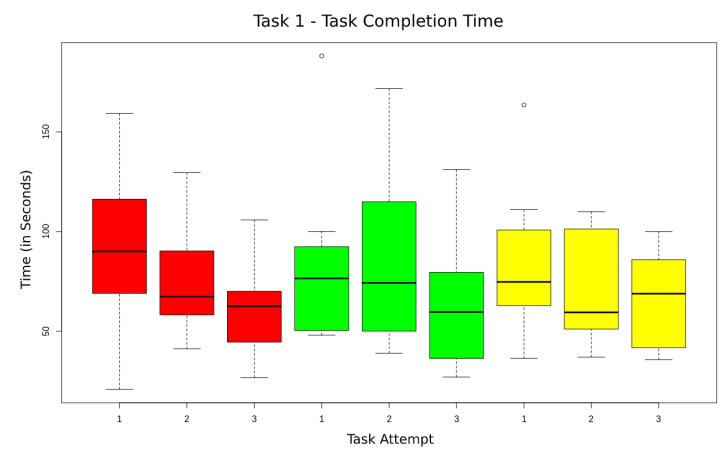

(a) Completion times for Task 1 (three runs) depending on the feedback method.

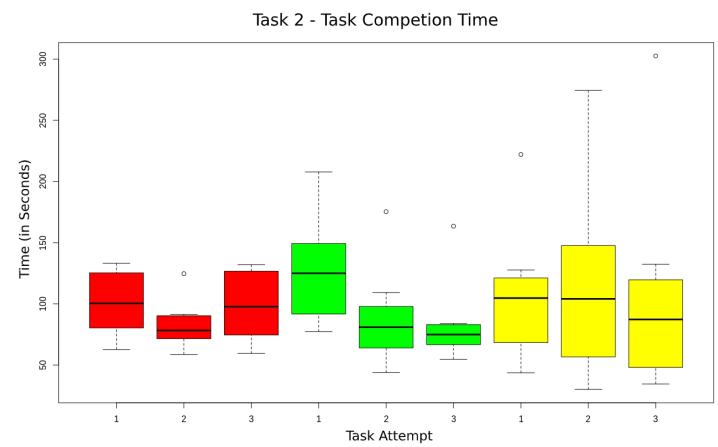

(b) Completion times for Task 2 (three runs) depending on the feedback method.

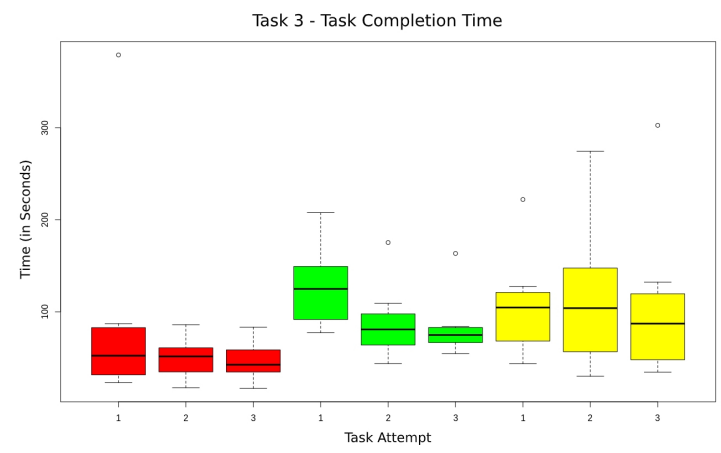

(c) Completion times for Task 3 (three runs) depending on the feedback method.

Fig. 4: Completion times for all tasks with no feedback (red), visual feedback (green), and vibration feedback (yellow). No significant differences are found between feedback methods across tasks or within each task. 


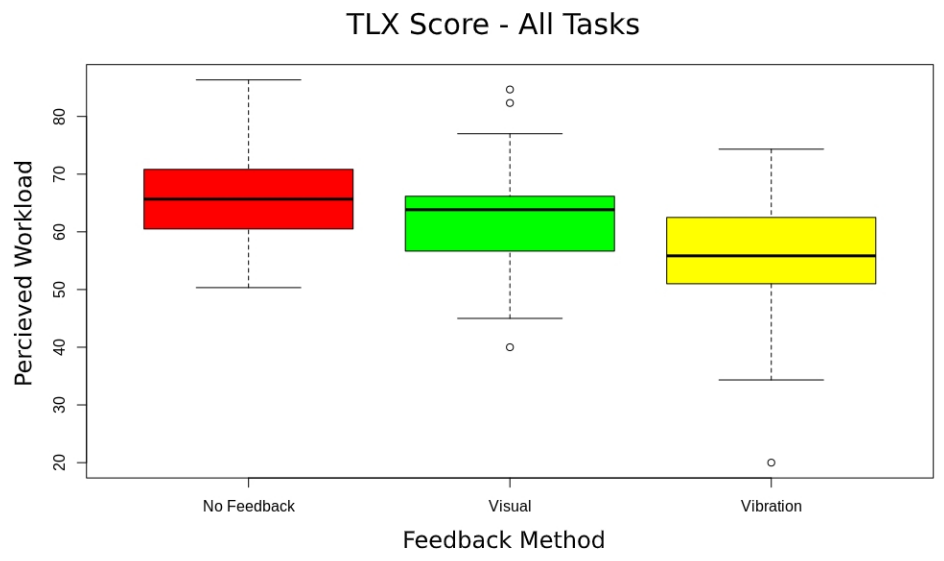

Fig. 5: Results from the NASA TLX perceived workload score for different feedback methods (across tasks). A significant reduction of perceived workload is found for vibration feedback.

[17] demonstrating similar results within a variation of tele-operation scenarios, providing the summary that the completion time between vibration feedback, stiffness feedback and no feedback was "statistically insignificant".

Regardless of this negative result in relation to the completion time, results present a learning effect within the tele-operation tasks. Across all tasks within the study it was found that participants were able to complete the tasks faster in their third attempt compared to their first attempt. This finding was investigated on the individual tasks; due to having a large amount of variance in the individual tasks, analysing the overall dataset was not possible. Two out of the three tasks investigated were considered to be significant findings with the final being a marginal, from this it is possible to conclude that there is a learning curve to the tele-operation scenarios and that the original hypothesis relating to this is correct. This finding also serves as validation of our general experimental procedure by demonstrating consistency of time measurement in relation to task performance. Hence, the lack of difference between feedback condition seems genuine, rather than being a potential artifact from flawed methodology.

Results analysis shows no significant findings in relation to the reduction in error rate across all tasks with the addition of vibration feedback. The analysis of error rates for visual cues is more intricate, as the parameterless test gives borderline significance, and the t-test must be considered inconclusive despite low p value due to the non-valid Gaussian assumption. While not fully conclusive, we believe that the difference found warrants attention and should be further investigated. A possible accuracy benefit from visual feedback to explain the observed gain could be the higher temporal resolution of peripheral vision, where the feedback is observed, compared to the temporal accuracy of sensing the onset vibration, which itself has a temporal extent. 
The most important finding within this research study is the effect vibration feedback has on reducing the overall perceived workload of a task. By using NASA-TLX [13] it was possible to analyse the individual tasks within the teleoperation study in relation to perceived workload. By implementing this over all 24 participants and across 72 tasks with the related feedback method, it is possible to cover all permutations of the study and in turn gain an understanding of the impact vibration feedback and visual cues have on a tele-operation task as a whole. From this analysis it is possible to confirm that vibration feedback significantly reduces the perceived workload of a tele-operation task, compared to no feedback, and more interestingly, compared to visual cues. Both findings presenting a significant result during analysis. This finding is important as it allows an understanding of the subtle impacts of such tactile sensory feedback and the positive influence that can be gained from the integration of sensory feedback systems. A plausible explanation for this finding could be a more efficient usage of attentional resources in the case of cross-modal cues [18], but which are still very incompletely understood for complex task performance [19]. Across the study there are limitations that could effect the strength of the results. The main limitation is that the study only addressed the early stages of previously untrained participants' learning process. Furthermore, each task was short and only run three times after an initial three minute familiarization.

A relevant alternative feedback method that was not addressed in this study concerns more directly mapped vibration feedback, with the sensor system being based on the gripper and the vibration system being mounted on the operators arm or hand (not a entirely natural sensation to feel extensive vibration in that area). If a system was to feature a direct mapping, for example a humanoid based robotic hand with a control glove there may be clearer results in relation to time of completion and error rate. Additionally the study used a static approach across all the tasks, with certain environmental variables remaining across the three tasks. These included the height of the table, the distance of the robot and the camera position. Keeping these throughout the tasks could allow for participants to gain familiarity with the environment and reduce the reliance on the feedback methods. Changing the environment dynamically could allow for further feedback effects.

Although the participant size was limited at 24, this study serves to highlight the effects of tactile feedback methods within a bilateral teleoperation scenario. The significant results and findings relating to the reduced perceived workload demonstrate that this study had sufficient power and that at least major differences in time to completion would have been detected if present. It can be speculated that the time of completion results may change with the increase of participant size, although there would be an expectation to see a level of consistency in the current results within a smaller dataset, similar to the reduced workload with the integration of vibration feedback. 


\section{Recommendations and Conclusions}

This paper presents the development of a tele-operation system with tactile sensing, and novel vibration or visual feedback. A study environment is developed to measure the impact of the tactile sensory feedback methods on multiple metrics with manipulation tasks. Results showing a significant reduction in perceived workload with the integration of vibration feedback, along with no finding relating to a reduction in task completion time and error rate. Based on the findings we are able to make the following recommendations. Due to results presenting a significant reduction in perceived workload with the addition of vibration feedback, we are able to make the recommendation of vibration feedback for use within tele-operation tasks that naturally require a high level of concentration or prolonged tasks.

The tasks undertaken within this study were relatively short, with task time ranging from 15.9 seconds - 302.6 seconds. By developing a further study that incorporates prolonged tasks could reflect an increased reduction in the overall workload of a task with the addition of vibration feedback. Furthermore, the study was made up of 20 out of 24 participants with no prior experience with teleoperation systems or robotics, by undertaking a similar study with experienced individuals there would be an assumed reduction in competition time, error rate and perceived workload. Prolonging the tele-operation tasks would allow for more in depth analysis of the impact of the tactile feedback systems on skilled performance rather than ongoing learning.

Although only partially statistically conclusive, the addition of visual cues showed a reduction in the error rate across all three tasks. From this we can recommend the use of visual feedback for tele-operation tasks that require a high level of accuracy. Future studies should investigate how the reduction in error rate can be translated into faster completion. Due to the inconclusive nature of vibration feedback relating to error rates, further studies should keep investigating both feedback methods. Further efforts should be made to investigate the role of cross-modal attention [19] in relation to the observed gains.

Most importantly, the results of this study highlight the need for broad assessment criteria within the evaluation of the tactile sensory feedback methods and the impact on tele-operation manipulation scenarios. The impact of feedback methods in tele-operation should not be investigated solely in terms of time of completion. Although for specific tasks, particular metrics should be analysed, the study highlights the need for a broader assessment. This study has utilized

perceived workload and error rates alongside completion time, which has allowed to uncover much more subtle implications of feedback than time alone.

\section{References}

1. Y. Liu, Semi-Autonomous Control of Multi-Robot Teams in Urban Search and Rescue Applications. PhD thesis, 2019. 
2. L. Fritsche, F. Unverzag, J. Peters, and R. Calandra, "First-person tele-operation of a humanoid robot," in 2015 IEEE-RAS 15th International Conference on Humanoid Robots (Humanoids), pp. 997-1002, IEEE, 2015.

3. W. J. Book, L. J. Love, and M. Farah, "A teleoperation testbed for nuclear waste restoration," Georgia Institute of Technology, 1994.

4. D. Ryu, C.-S. Hwang, S. Kang, M. Kim, and J.-B. Song, "Wearable haptic-based multi-modal teleoperation of field mobile manipulator for explosive ordnance disposal," in IEEE International Safety, Security and Rescue Rototics, Workshop, 2005., pp. 75-80, IEEE, 2005.

5. S. Kim, C. H. Kim, Y. C. Seo, S. H. Jung, G. S. Lee, and B. S. Han, "Development of tele-operated mobile robot in nuclear power plants," IFAC Proceedings Volumes, vol. 34, no. 4, pp. 239-244, 2001.

6. Y. Nagai, S. Tsuchiya, T. Iida, and S. Kimura, "Audio feedback system for teleoperation experiments on engineering test satellite vii system design and assessment using eye mark recorder for capturing task," IEEE Transactions on Systems, Man, and Cybernetics-Part A: Systems and Humans, vol. 32, no. 2, pp. 237-247, 2002.

7. J. Dennerlein, R. Howe, E. Shahoian, and C. Olroyd, "Vibrotactile feedback for an underwater telerobot," in Robotics and applications; Robotic and manufacturing systems recent results in research, development and applications International symposium; 8th, pp. 244-249, Citeseer, 2000.

8. V. Hayward, O. R. Astley, M. Cruz-Hernandez, D. Grant, and G. Robles-De-LaTorre, "Haptic interfaces and devices," Sensor review, 2004.

9. P. G. De Barros, R. W. Lindeman, and M. O. Ward, "Enhancing robot teleoperator situation awareness and performance using vibro-tactile and graphical feedback," in 2011 IEEE Symposium on 3D User Interfaces (3DUI), pp. 47-54, IEEE, 2011.

10. A. M. Okamura, "Haptic feedback in robot-assisted minimally invasive surgery," Current opinion in urology, vol. 19, no. 1, p. 102, 2009.

11. M. M. Alex, "Evaluating the effects of haptic and visual feedback on the teleoperation of remote robots,"

12. D. Whitney, E. Rosen, E. Phillips, G. Konidaris, and S. Tellex, "Comparing robot grasping teleoperation across desktop and virtual reality with ros reality," in Robotics Research, pp. 335-350, Springer, 2020.

13. N. H. P. R. Group et al., "Task load index (nasa-tlx) v1. 0 computerised version," NASA Ames Research Centre, 1987.

14. H. Reddivari, C. Yang, Z. Ju, P. Liang, Z. Li, and B. Xu, "Teleoperation control of baxter robot using body motion tracking," in 2014 International conference on multisensor fusion and information integration for intelligent systems (MFI), pp. 1-6, IEEE, 2014.

15. S. Pfeiffer, "Vive teleop stuff," Mar 2018. GitHub: https://github.com/uts-magiclab/htc_vive_teleop_stuff.

16. "Hy-srf05: Srf05 technical documentation." https://www.robotelectronics.co.uk/htm/srf05tech.htm.

17. A. Casqueiro, D. Ruivo, A. Moutinho, and J. Martins, "Improving teleoperation with vibration force feedback and anti-collision methods," in Robot 2015: Second Iberian Robotics Conference, pp. 269-281, Springer, 2016.

18. J. Driver and C. Spence, "Cross-modal links in spatial attention," Philosophical Transactions of the Royal Society of London. Series B: Biological Sciences, vol. 353, no. 1373 , pp. 1319-1331, 1998.

19. T. K. Ferris and N. B. Sarter, "Cross-modal links among vision, audition, and touch in complex environments," Human Factors, vol. 50, no. 1, pp. 17-26, 2008. 